\title{
Monte Carlo Approach to Total $\pi$-Electron Energy of Conjugated Hydrocarbons
}

\author{
I. Gutman and M. Rašković \\ Faculty of Science, University of Kragujevac
}

Z. Naturforsch. 40 a, 1059-1061 (1985); received July 3, 1985

Approximating the $\pi$-electron molecular orbital energy levels by uniformly distributed random variables, a McClelland-type formula for the total $\pi$-electron energy is obtained. Conditions are determined under which a given distribution function will result in a formula of McClelland type.

The total $\pi$-electron energy $(E)$, as calculated within the Hückel molecular orbital model, has for a long time been attracting the attention of theoretical chemists [1, 2]. Especially important seems to be the recent finding [2] that a precise linear relationship exists between $E$ and the kinetic energy of the $\pi$-electrons.

In the following the conjugated molecule under consideration will be represented by its molecular graph [3]. This graph has $n$ vertices and $m$ edges. For the sake of simplicity we shall assume that the molecule considered is an alternant hydrocarbon and that $n$ (= the number of carbon atoms) is an even number. If $x_{1}, x_{2}, \ldots, x_{n}$ are the eigenvalues [3] of the molecular graph, labeled in a non-increasing order, then

$$
E=2 \sum_{j=1}^{n / 2} x_{j}
$$

A relation which will be needed in the forthcoming discussion is [3]

$$
\sum_{j=1}^{n / 2} x_{j}^{2}=m
$$

Now, if $x_{1}, x_{2}, \ldots, x_{n / 2}$ are viewed as random numbers, that is $n / 2$ independent realizations of a random variable $X$, then their sample mean $e(X)$ and sample dispersion $d(X)$ are given by

$$
\begin{aligned}
& e(X)=\frac{1}{n / 2} \sum_{j=1}^{n / 2} x_{j}=E / n, \\
& d(X)=\frac{1}{n / 2} \sum_{j=1}^{n / 2}\left[x_{j}-e(X)\right]^{2}=2 m / n-(E / n)^{2} .
\end{aligned}
$$

Reprint requests to Prof. Dr. Ivan Gutman, Faculty of Science, P.O. Box 60, YU-34000 Kragujevac, Yugoslavia.
Since obviously $d(X) \geqq 0$, we get from (4)

$$
E \leqq \sqrt{2 m n},
$$

which is just the McClelland upper bound for the total $\pi$-electron energy [4].

In the same paper [4] in which the upper bound (5) has been deduced, it has been established that $E$ can be fairly well reproduced by the expression

$$
E=a \sqrt{2 m n},
$$

where $a$ is an empirical parameter, whose value is about 0.9 . The accuracy of (6) was verified in [5], where a comparative study of a great number of approximate topological formulas has been performed.

It has been shown [6] that the upper bound (5) is related to the assumption that all the (positive) graph eigenvalues are degenerate. Namely, the following result was deduced in [6]:

Lemma 1. Under the constraint (2), the greatest possible value of $E$ is $(2 m n)^{1 / 2}$, and this maximum is reached if and only if $x_{1}=x_{2}=\cdots=x_{n / 2}$.

On the basis of Lemma 1 one may be inclined to expect that the validity of the approximation (6) is also stipulated by a relatively small deviation of graph eigenvalues from their mean value. Surprisingly, however, if the opposite is true, namely if all eigenvalues are mutually distinct, an expression for $E$, closely similar to (6) exists [6]:

Lemma 2. If the numbers $x_{j}$ are equidistant, that is $x_{j+1}-x_{j}=$ const for all $j=1,2, \ldots, n / 2-1$, then under the constraint (2),

$$
E=a(n) \sqrt{2 m n},
$$

where the function $a(n)=\left[3 n^{2} /\left(4 n^{2}-4\right)\right]^{1 / 2}$ rapidly converges to $(3 / 4)^{1 / 2}=0.87$ when $n$ tends to infinity. 
Comparing Lemmas 1 and 2 we see that quite different distributions of the graph eigenvalues may lead to McClelland-type formulas for the total $\pi$ electron energy. In the present paper further arguments in favour of this conclusion will be given. For the beginning we formulate the following result.

Le mma 3. If $x_{1}, x_{2}, \ldots, x_{n / 2}$ are random numbers, uniformly distributed in the interval $(0, p)$, then for an arbitrary positive value of $p$,

$$
\langle E\rangle=b \sqrt{2\langle m\rangle n},
$$

where $b=(3 / 4)^{1 / 2}=0.87$ equals the limit value of the function $a(n)$ in (7). In (8), $\langle E\rangle$ and $\langle m\rangle$ denote the expectation values of $E$ and $m$, as defined via (1) and (2).

\section{Numerical work}

In order to test Lemma 3 we have chosen the interval $(0, p)$ by generating a random number $r \in(0,1)$ and setting $p=1 / r$. Then by generating the random numbers $r_{j} \in(0,1), j=0,1,2, \ldots$ we defined $n / 2=\left[50 r_{0}\right]$ and $x_{j}=p r_{j}, j=1,2, \ldots, n / 2$ and calculated $E$ and $m$ by means of (1) and (2). After repeating this procedure 20 times (for a fixed value of $p$ ), we determined the parameter $b$ in (8) by least squares fitting.

In Table 1 are presented ten typical results obtained in this manner.

\section{Proof of Lemma 3}

Suppose that $X$ is a continuous random variable, uniformly distributed in the interval $(0, p)$. Then its distribution function $\varrho(x)$ is equal to $1 / p$. Bearing in mind (1) and (2), we have

$$
\begin{aligned}
& \langle E\rangle=(n / 2) \int_{0}^{p} 2 x \varrho(x) \mathrm{d} x=n p / 2, \\
& \langle m\rangle=(n / 2) \int_{0}^{p} x^{2} \varrho(x) \mathrm{d} x=n p^{2} / 6 .
\end{aligned}
$$

Elimination of the parameter $p$ from (9) and (10) results immediately in (8). Note the very good agreement between the parameter $b$ in (8) and the empirically determined value for $a$ in McClelland's formula (6).

A more careful examination of the above proof shows that the assumption of the uniform distribu- tion of the variable $X$ is not at all necessary for the derivation of a McClelland-type formula for $E$.

Indeed, let $X$ be a continuous random variable whose values lie in the interval $(0, p)$ and whose distribution function is $\varrho(x)$. Suppose that the first and the second moment of $X$ are proportional to $p$ and $p^{2}$, respectively:

$$
\begin{aligned}
& \int_{0}^{p} x \varrho(x) \mathrm{d} x=c_{1} p, \\
& \int_{0}^{p} x^{2} \varrho(x) \mathrm{d} x=c_{2} p^{2},
\end{aligned}
$$

where $c_{1}$ and $c_{2}$ are constants, independent of $p$. Then, according to (9) and (10),

$$
\langle E\rangle=n c_{1} p
$$

and

$$
\langle m\rangle=n c_{2} p^{2} / 2,
$$

and therefore

$$
\langle E\rangle=c \sqrt{2\langle m\rangle n},
$$

where

$$
c=c_{1} c_{2}^{-1 / 2} .
$$

Clearly, the distribution function $\varrho(x)$ must depend in a pertinent way on the parameter $p$.

We show now that for an arbitrary function $f(x)$ which is non-negative in the interval $(0,1)$ and whose integral

$$
I=\int_{0}^{1} f(x) \mathrm{d} x
$$

Table 1. Monte Carlo estimation of the parameter $b$ in (8). Each result is obtained by a least squares fitting of data for 20 "random molecules".

\begin{tabular}{rll}
\hline No. & $b$ & $\begin{array}{l}\text { Correlation } \\
\text { coefficient }\end{array}$ \\
\hline 1 & 0.888 & 0.9995 \\
2 & 0.879 & 0.99990 \\
3 & 0.863 & 0.99990 \\
4 & 0.854 & 0.998 \\
5 & 0.862 & 0.9997 \\
6 & 0.904 & 0.998 \\
7 & 0.828 & 0.9998 \\
8 & 0.855 & 0.9990 \\
9 & 0.835 & 0.9997 \\
10 & 0.882 & 0.998 \\
\hline
\end{tabular}


exists, we can choose the distribution function $\varrho(x)$ as

$$
\varrho(x)=(I p)^{-1} f(x / p),
$$

and $\varrho(x)$ has the properties (11) and (12). As a matter of fact, for a distribution function of the form (18), the $k$-th moment of $X$ is proportional to $p^{k}$. This can be easily seen by direct calculation of the $k$-th moment:

$$
\int_{0}^{p} x^{k} \varrho(x) \mathrm{d} x=\left(p^{k} / I\right) \int_{0}^{1} y^{k} f(y) \mathrm{d} y,
$$

where $y=x / p$. Hence

$$
\begin{aligned}
& c_{1}=\int_{0}^{1} x f(x) \mathrm{d} x / \int_{0}^{1} f(x) \mathrm{d} x, \\
& c_{2}=\int_{0}^{1} x^{2} f(x) \mathrm{d} x / \int_{0}^{1} f(x) \mathrm{d} x,
\end{aligned}
$$

and we have arrived at the following result:

Theorem 1. Let $f(x)$ be a function having nonnegative values in the interval $(0,1)$, whose integral (17) exists. If $x_{1}, x_{2}, \ldots, x_{n / 2}$ are random numbers, distributed in the interval $(0, p)$ according to (18), then for an arbitrary positive value of $p$, a McClel-

[1] For recent work on total $\pi$-electron energy see: I. Gutman, A. V. Teodorović, and Lj. Nedeljković, Theor. Chim. Acta 65, 23 (1984); L. Türker, Match (Mülheim) 16, 83 (1984); Y. Jiang, A. Tang, and R. Hoffmann, Theoret. Chim. Acta 66, 183 (1984).

[2] H. Ichikawa and Y. Ebisawa, J. Amer. Chem. Soc. 107, 1161 (1985).

[3] For details of the topological theory of conjugated molecules and fundamentals of graph theory see: A. Graovac, I. Gutman, and N. Trinajstić, Topological land-type relation (15) exists between $\langle E\rangle,\langle m\rangle$ and $n$, where the multiplyer $c$ is determined via (16), (20) and (21).

Choosing $f(x)=1$, the above theorem reduces to Lemma 3.

Theorem 1 shows that the existence of a McClelland-type dependency between the total $\pi$-electron energy and the topological parameters $n$ and $m$ is almost independent of the assumed distribution of graph eigenvalues. This also explains the success of the approximate topological formula (6) [5]. The distribution of graph eigenvalues influences, however, the value of the empirical constant $a$. The fact that the parameters $a$ in (6) and $b$ in (8) have very close numerical values indicates that the assumptions made in Lemma 3 are not very far from the reality.

\section{Acknowledgement}

One of the authors (I.G.) was an Alexander von Humboldt fellow in 1985. He thanks the Alexander von Humboldt Foundation for financial support and the staff of the Max-Planck-Institut für Strahlenchemie in Mülheim/Ruhr for assistance and hospitality.

Approach to the Chemistry of Conjugated Molecules, Springer-Verlag, Berlin 1977; I. Gutman and O. E. Polansky, Mathematical Aspects in Organic Chemistry, Springer-Verlag, Berlin, to appear.

[4] B. J. McClelland, J. Chem. Phys. 54, 640 (1971).

[5] I. Gutman, Lj. Nedeljković, and A. V. Teodorović, Bull. Soc. Chim. Beograd 48, 495 (1983); I. Gutman, A. V. Teodorović, and Ž. Bugarčić, Bull. Soc. Chim. Beograd 49, 521 (1984).

[6] I. Gutman, Match (Mülheim) 14, 71 (1983). 